\title{
The effect of population subdivision on estimates of the likelihood ratio in criminal cases using single-locus DNA probes
}

\author{
JOHN BROOKFIELD \\ Department of Genetics, University of Nottingham, Queens Medical Centre, Nottingham NG7 2UH, U.K.
}

\begin{abstract}
The interpretation of single-locus DNA profile evidence in criminal cases in normally performed using an assumption of independence of frequencies of alleles at different loci. The critical parameter is the probability of a suspect matching a criminal's DNA profile by chance. Many authors have been concerned that the assumption of linkage equilibrium may be inaccurate and that the strength of evidence against suspects may be thereby exaggerated. Some have calculated the maximum size of this effect. What matters, however, is not the upper bound of what the true probability might be, but what the actual probability is in a subdivided population. By calculating this, one can show that the effect of population subdivision on the strength of DNA profile evidence is very minor.
\end{abstract}

Keywords: DNA profiling, forensic science, likelihood ratios, linkage disequilibrium, minisatellites, population genetics.

\section{Introduction}

In many criminal cases, DNA is extracted from a blood or semen stain found at the scene of the crime and blots of the DNA are sequentially probed with a series of minisatellite probes (Wong et al., 1986, 1987). These probes reveal individual minisatellite loci, and thus normally two heterozygous bands or alleles. The profiles thus generated can be compared with those from a suspect, and, if a match is obtained, this constitutes evidence against him. The strength of the evidence is given by the likelihood ratio, which is the probability of obtaining a match if the DNAs are derived from the same source, divided by the probability if the DNAs are from different sources. As the first probability is essentially one, the crucial parameter is the probability of a chance match. If $k$ probes are used and thus $k$ genetic loci detected, and if all are heterozygous in the scene-of-crime stain, then the probability that a random man from the population would match by chance is normally calculated to be

$$
2^{k} \prod_{i=1}^{2 k} p_{i}
$$

Here the $p_{i}$ values are the population frequencies of the $2 k$ alleles observed in the scene-of-crime profile. They correspond not to well-defined allelic frequencies but rather the summed population frequencies of bands which fall sufficiently close to the scene-of-crime profile bands to be declared to match. The width of the window of bands thus included depends on the measurement error of band sizes. This equation thus assumes that alleles are independent, i.e. in linkage equilibrium. Many authors [e.g. Lander (1989), Cohen (1990) and Nicholls \& Balding (1991)] have pointed out that if the population is divided into subpopulations, the probability calculated using equation (1) will usually be too small and thus the suspect may be falsely incriminated. This is undoubtably true but the important statistic is the quantitative effect of this problem, particularly in an environment in which matches are far more likely to arise from laboratory errors than from true chance matches between individuals (Hagerman 1990). Cohen (1990) attempts to quantify the strength of this effect by choosing extreme values for subpopulation allelic frequency variation and finding that this makes frequency calculations performed using equation (1) inaccurate by many orders of magnitude. This is not helpful because equation (1) can clearly be made arbitrarily inaccurate by simple imagining populations which diverge to increasing degrees from the assumption of independence which equation (1) makes. What is required is a serious attempt 
to calculate the effect on the accuracy of equation (1) of realistic degrees of population substructure. Nicholls \& Balding (1991) make a useful contribution to this task by considering population subdivision in terms of $F_{\mathrm{ST}}$ values. They point out that if one considers a single locus with two rare alleles with frequency 0.01 but with an $F_{\mathrm{ST}}$ value of 0.05 , then the probability of a match will be given by

$2\left[p_{\mathrm{f}}+F_{\mathrm{ST}}\left(1-p_{\mathrm{f}}\right)\right]\left[p_{\mathrm{m}}+F_{\mathrm{ST}}\left(1-p_{\mathrm{m}}\right)\right]$

where $p_{\mathrm{f}}$ and $p_{\mathrm{m}}$ are the frequencies in the population as a whole of alleles derived from father and mother respectively. The inclusion of a 0.05 value for $F_{\mathrm{ST}}$, and 0.01 for $p_{\mathrm{f}}$ and $p_{\mathrm{m}}$ causes a 35 -fold change in the calculated probability relative to that if $F_{\mathrm{ST}}$ was 0.0 . This is at a single locus and other loci will further increase the ratio. These authors suggest that, because it is possible that the criminal and suspect are both drawn from the same subpopulation, in which case they would show such a high $F_{\mathrm{ST}}$ value, then a probability calculated in this way, which would be an upper bound of the true probability, should be used for conservativeness. It is, however, a misidentification of the meaning of a probability, which, by its nature, is an encapsulation of partial knowledge, to use as a total probability the probability that would be associated with an event which itself is highly improbable. There really is no point in calculating what an upper bound of a probability might be. The important parameter is to calculate the actual probability of a chance match in a subdivided population. To do this, it is clear that equation (2) with the $p$ and $F_{\mathrm{ST}}$ figures given is, in itself, inadequate. There are three reasons. Firstly, the value of 0.01 for $p_{\mathrm{f}}$ and for $p_{\mathrm{m}}$ is very low, much lower than would normally be used in forensic work. Secondly, the $F_{\text {ST }}$ value of 0.05 is absurdly high: 0.01 or 0.02 are much more reasonable values. Finally, the equation assumes that the true criminal and suspect are drawn from the same subpopulation, which may a priori be unlikely. The model must thus be modified to give us realistic probabilities in a subdivided population.

Table 1 Relationship between likelihood ratio, $F_{\mathrm{ST}}$ and the number of subpopulations for different band frequencies

\begin{tabular}{|c|c|c|c|c|c|}
\hline & \multicolumn{5}{|c|}{ Number of subpopulations } \\
\hline & 2 & 5 & 10 & 20 & 50 \\
\hline \multicolumn{6}{|c|}{$\begin{array}{r}p_{\mathrm{s}}=0.02,0.03 \\
\\
0.04,0.05\end{array}$} \\
\hline$F_{\mathrm{ST}} 0.00$ & $4.34 \times 10^{10}$ & & & & \\
\hline 0.01 & $1.02 \times 10^{10}$ & $2.06 \times 10^{10}$ & $2.82 \times 10^{10}$ & $3.46 \times 10^{10}$ & $3.94 \times 10^{10}$ \\
\hline 0.02 & $1.96 \times 10^{9}$ & $4.78 \times 10^{9}$ & $8.78 \times 10^{9}$ & $1.47 \times 10^{10}$ & $2.45 \times 10^{10}$ \\
\hline 0.03 & - & $1.25 \times 10^{9}$ & $2.46 \times 10^{9}$ & $4.68 \times 10^{9}$ & $1.01 \times 10^{10}$ \\
\hline 0.04 & - & $3.96 \times 10^{8}$ & $7.87 \times 10^{8}$ & $1.55 \times 10^{8}$ & $3.69 \times 10^{9}$ \\
\hline 0.05 & - & $1.38 \times 10^{8}$ & $2.75 \times 10^{8}$ & $5.48 \times 10^{8}$ & $1.35 \times 10^{9}$ \\
\hline \multicolumn{6}{|c|}{$\begin{array}{r}p_{\mathrm{s}}=0.06,0.07 \\
0.08,0.09\end{array}$} \\
\hline$F_{\mathrm{ST}} 0.00$ & $6.84 \times 10^{7}$ & & & & \\
\hline 0.01 & $4.67 \times 10^{7}$ & $6.01 \times 10^{7}$ & $6.43 \times 10^{7}$ & $6.64 \times 10^{7}$ & $6.76 \times 10^{7}$ \\
\hline 0.02 & $2.23 \times 10^{7}$ & $4.06 \times 10^{7}$ & $5.18 \times 10^{7}$ & $5.91 \times 10^{7}$ & $6.44 \times 10^{7}$ \\
\hline 0.03 & $1.05 \times 10^{7}$ & $2.31 \times 10^{7}$ & $3.53 \times 10^{7}$ & $4.69 \times 10^{7}$ & $5.79 \times 10^{7}$ \\
\hline 0.04 & $5.24 \times 10^{6}$ & $1.25 \times 10^{7}$ & $2.15 \times 10^{7}$ & $3.30 \times 10^{7}$ & $4.80 \times 10^{7}$ \\
\hline 0.05 & $2.76 \times 10^{6}$ & $6.76 \times 10^{6}$ & $1.25 \times 10^{7}$ & $2.14 \times 10^{7}$ & $3.65 \times 10^{7}$ \\
\hline \multicolumn{6}{|l|}{$p_{\mathrm{s}}=0.15$} \\
\hline$F_{\mathrm{ST}} 0.00$ & 243,843 & & & & \\
\hline 0.01 & 223,614 & 238,039 & 241,138 & 242,601 & 243,368 \\
\hline 0.02 & 177,841 & 220,167 & 232,396 & 238,265 & 241,604 \\
\hline 0.03 & 130,531 & 192,382 & 217,014 & 230,149 & 238,265 \\
\hline 0.04 & 92,505 & 159,769 & 195,695 & 217,912 & 232,937 \\
\hline 0.05 & 65,660 & 127,258 & 170,213 & 201,450 & 225,225 \\
\hline
\end{tabular}




\section{Calculation of the likelihood ratio in a subdivided population}

Let us suppose that the population consists of $n$ subpopulations of equal size, and that we examine the population using $k$ probes. Let us further suppose that, while the probes may detect different levels of heterozygosity, at any locus, all the alleles at a locus have the same (low) frequency. Thus $p_{\mathrm{i}}$ is the frequency in the population of any allele at locus $i$. Now we have a scene-of-crime profile, which we assume for simplicity is completely heterozygous, and we have a suspect who matches at all $k$ loci tested. The probability of this happening will depend upon whether the source of the scene-of-crime profile and the suspect come from the

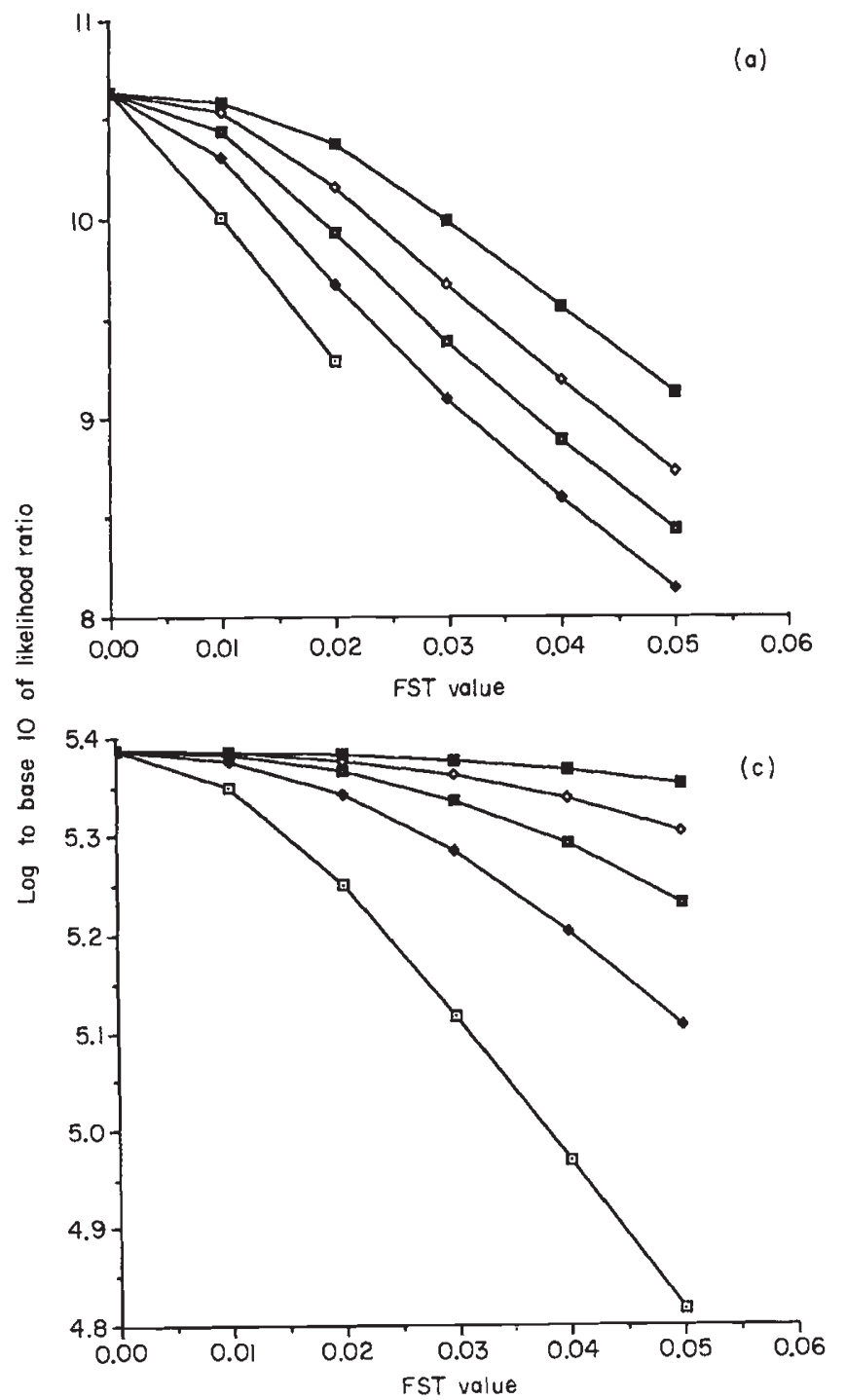

same subpopulation. The probability that they do will be $1 / n$ and the probability that they do not will be $(n-1) / n$. The probability of a chance match if they are from the same subpopulation, $P_{s}$, will be rather easy to calculate from Nicholls and Balding's formula. It will be

$P_{\mathrm{s}}=2^{k} \prod_{i=1}^{k}\left(p_{i}+F_{\mathrm{ST}}-p_{i} F_{\mathrm{ST}}\right)^{2}$

If the two individuals are from different subpopulations then note that because the frequency of an allele in a subpopulation in which it is common is $\left(p_{i}+F_{\mathrm{ST}}-p_{i} \cdot F_{\mathrm{ST}}\right)$, and $p_{i}$ is a mean across subpopula-

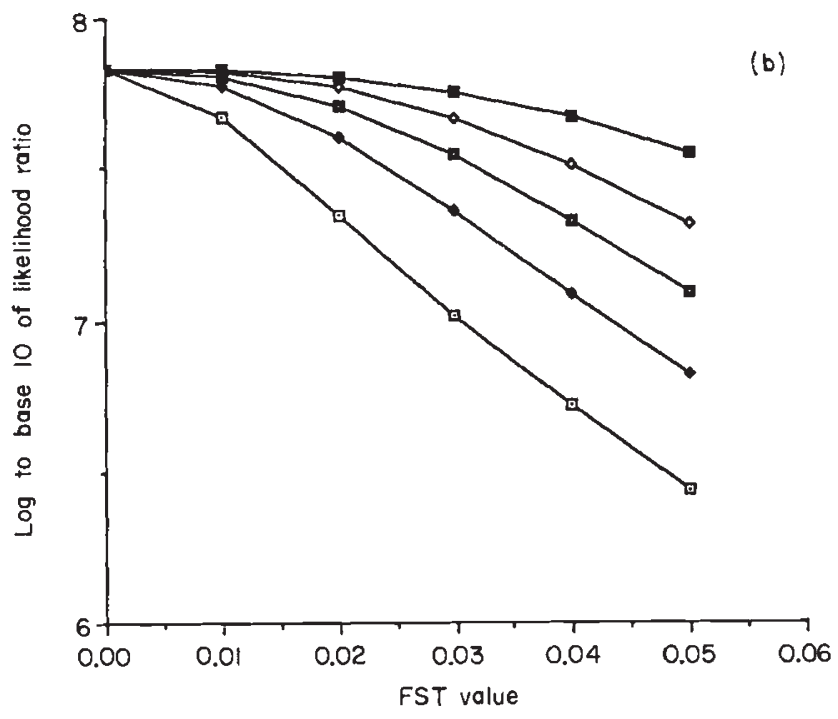

Fig. 1 The relationship between the logarithm to base 10 of the likelihood ratio and the $F_{\mathrm{ST}}$ value for various values of $n$ using three distributions of allelic frequencies. (a) $p_{1}=0.02, p_{2}=0.03, p_{3}=0.04$, and $p_{5}=0.05$; (b) $p_{1}=0.06, p_{2}=0.07, p_{3}=0.08$, and $p_{4}=0.09 ;$ (c) $p_{1}=p_{2}=p_{3}=p_{4}=0.15$. There is very little proportional effect on the likelihood ratio as a result of population subdivision, particularly when the $n$ value is high, $F_{\mathrm{ST}}$ values are reasonably small, and the likelihood ratio without subdivision is quite small. Subpopulations: $(\varpi) 2,(\diamond) 5,(\square) 10,(\diamond) 20,(\bullet) 50$. 
tions (from the way in which it is measured), then the frequency of an allele in a population in which it is rare, which we will call $p_{r i}$, will be

$\left[\left(n+F_{\mathrm{ST}}-1\right) p_{i}-F_{\mathrm{ST}}\right] /(n-1)$.

This constraint thus requires $n$ to be equal to or bigger than

$\left(p_{i}+F_{\mathrm{ST}}-p_{i} F_{\mathrm{ST}}\right) / p_{i}$.

The probability of a chance match at all $k$ loci if the suspect and the source of the scene-of-crime stain are from different subpopulations, $P_{\mathrm{d}}$, will be given by

$$
P_{\mathrm{d}}=2^{k} \prod_{i=1}^{k} p_{r i}^{2}
$$

and thus the total probability of a chance match by $\left[P_{\mathrm{s}}+(n-1) P_{\mathrm{d}}\right] / n$. As a match is certain if the suspect is the source of the scene-of-crime DNA, the likelihood ratio, which is the probability of the data given that the DNA sources are the same, divided by the probability of the data if the DNA sources are different, will be the reciprocal of the probability of a chance match. Table 1 gives the results of calculating the effects of subdivision as a function of $n$ and $F_{\mathrm{ST}}$ in three situations, differing in the band frequencies. These results are shown in Fig. $1(\mathrm{a}-\mathrm{c})$. Firstly, we see the situation in which the four loci have, respectively, band frequencies of $0.02,0.03$, 0.04 , and 0.05 . In this case a match would produce a likelihood ratio of 43 billion assuming no subdivision, which would be reduced around 300 -fold in the $F_{\mathrm{ST}}=0.05, n=5$ case. A 300 -fold reduction would seem a large effect, but two things must be noted. Firstly, $F_{\mathrm{ST}}=0.05$ is unreasonably large, and $n=5$ unreasonably small. More realistic values of $F_{\mathrm{ST}}=0.02$ and $n=10$ give only a fivefold reduction in the likelihood ratio. Secondly, 138 million is still a very large likelihood ratio, which would be seen to be definitive in a criminal case.

The most important aspect of the data, however, can be revealed by looking at larger band frequencies. Equation (3) above shows that the effect of population subdivision depends critically on the relative sizes of $p_{i}$ and $F_{\mathrm{ST}}$, so that if $p_{i}$ values become larger, population subdivision has a much smaller effect. Thus, when $p_{i}$ values are $0.06,0.07,0.08$, and 0.09 , the likelihood ratio of 68 million calculated, assuming no subdivision, is reduced only 25 -fold with the most extreme values of subdivision, and with our $n=10, F_{\mathrm{ST}}=0.02$ case, is reduced by only 24 per cent. Finally, with all $p_{i}$ equal to 15 per cent, which gives 244 thousand as a likelihood ratio, the biggest reduction we see is less than fourfold and a more probable one is the 5 per cent reduction to 232 thousand. It is impossible to find a case in which a likelihood ratio constituting strong evidence against a subject is converted to one in which the evidence has become weak.

Throughout this analysis it is assumed that DNA profiles are examined using the concept of a match, in which the divergence in mobility between the suspect and scene-of-crime DNAs is assessed as to whether it falls within some predefined window of mobility differences, with the window size determined by the experimental error. The concept of a match could, however, be avoided, following Evett et al. (1990), by using a likelihood ratio of the probabilities of the precise degrees of separation between the suspect and sceneof-crime DNAs, in which the numerator is calculated from normal distributions of measurement error, and the denominator from the population distributions of allelic size measurements.

The likelihood ratio thus calculated is a ratio between two very small probabilities, but the calculations outlined here are still of relevance to the denominator. However, my conclusions above the subdivision only significantly reduce the likelihood ratio if the allelic frequencies are small and thus the likelihood ratio large, is weakened by such an analysis because it is possible that cases exist in which the denominator could be very small and thus subject to a major subdivision effect. The numerator, however, is also very small because the suspect's bands fall some way away from the scene-of-crime profile bands. The likelihood ratio could thus be fairly small but nevertheless affected by population subdivision.

\section{References}

COHEN, J. E. 1990. DNA fingerprinting for forensic identification: potential effects on data interpretation of subpopulation heterogeneity and band number variability. Am. J. Hum. Genet., 46, 358-368.

EVETT, I. W., WERRETT, D. J., PINCHIN, R. AND GILL, P. 1990. Bayesian analysis of single locus DNA profiles. In: The International Symposium on Human Identification 1989. Promega Corporation, Madison, W.I. pp. 77-101.

HAGERMAN, P. J. 1990. DNA typing in the forensic area. Am. J. Hum. Genet., 47, 876-877.

LANDER, E. S. 1989. DNA fingerprinting on trial. Nature, 339, 501-505.

NICHOLLS, R. A. AND BALDING, D. J. 1991. Effects of population structure on DNA fingerprinting analysis in forensic science. Heredity, 66, 297-302.

WONG, Z., WILSON, V., PATEL, I., POVEY, S. AND JEFFREYS, A. J. 1986. Cloning a selected fragment from a human DNA 'fingerprint': isolation of an extremely polymorphic minisatellite. Nucl. Acids Res., 14, 4605-4616.

WONG, Z., WILSON, V., PATEL, I., POVEY, S. AND JEFFREYS, A. J. 1987. Characterisation of a panel of highly variable minisatellites cloned from human DNA. Ann. Hum. Genet., 51, 259-288. 